\title{
Archetype of Olfactory and Gustatory Dysfunction in Breakthrough COVID-19 Illness
}

\author{
$\operatorname{Prem}_{\text {Sagar }}^{1}$ (D) Rajeev Kumar ${ }^{1} \cdot$ Alok Thakar $^{1}$
}

Received: 26 August 2021/Accepted: 18 November 2021/Published online: 25 November 2021

(C) Association of Otolaryngologists of India 2021

\begin{abstract}
Vaccination against SARS CoV-2 has resulted in COVID-19 illness with reduced incidence and milder symptomatology. With the intent to analyze the olfactory and gustatory disturbances in breakthrough COVID-19 illness, we conducted a prospective observational study on a cohort of COVID-19 positive symptomatic otolaryngologist physicians. These doctors being well sensitized to the symptoms and signs of loss of smell and taste, reported even very mild and transient symptoms. This cohort of 25 physicians included 16 unvaccinated and 9 vaccinated otolaryngologists. The overall incidence of smell and taste disturbances reported in this cohort was $80 \%$ and $84 \%$ respectively. The incidence of olfactory dysfunction was $81 \%$ in the unvaccinated group and $78 \%$ in the vaccinated group. Similarly, the incidence of gustatory dysfunction was $81 \%$ in the unvaccinated group and $89 \%$ in the vaccinated group. However, the incidence of complete loss of smell was $54 \%$ in the unvaccinated group and $100 \%$ in the vaccinated group. Ageusia was noted in $15 \%$ of the unvaccinated physicians as compared to $25 \%$ of the vaccinated ones. The average duration of smell and taste loss were lesser in the physicians who were vaccinated with two doses than their unvaccinated counterparts. Larger studies are desirable to add further knowledge on the equal incidences and higher degree of loss of smell and taste observed in breakthrough COVID-19 illness as observed in our small prospective cohort study.
\end{abstract}

Prem Sagar

sagardrprem@gmail.com

1 Department of Otolaryngology and Head Neck Surgery, AIIMS, New Delhi 110029, India
Keywords COVID-19 - Vaccination .

Chemosensory dysfunction - Loss of smell - Loss of taste

\section{Introduction}

In the Indian subcontinent, vaccination of country's frontline health workers against SARS CoV-2 started at the dawn of this year. In COVID-19 illness, lower incidence and milder severity of symptomatology has been noted in the post-vaccination population as compared to the nonvaccinated group $[1,2]$. We prospectively observed the incidence and severity of olfactory and gustatory dysfunction in a cohort of otolaryngologist physicians who were symptomatic with COVID-19 infection from June 2020 to June 2021 in a tertiary care institute in north India.

\section{Methods}

This cohort was restricted to otolaryngologists who were aware of the symptoms and signs of loss of smell and taste and hence were able to report even very mild and transient symptomatology. During the first wave (June-Nov 2020), we reported on universal occurrence and complete recovery of olfactory and gustatory dysfunction in six otolaryngologists [3]. None of these physicians had had vaccination against COVID-19 as vaccination had not been introduced in the country till then. During the second wave (April-May 2021), we recruited 19 more otolaryngologists and tracked their chemosensory dysfunction by regular telephonic review till complete recovery or minimum up to 12 weeks. 
Table 1 Details of olfactory and gustatory dysfunction in the unvaccinated $(\mathrm{V}-$ )and vaccinated $(\mathrm{V}+)$

\begin{tabular}{|c|c|c|c|c|c|c|}
\hline \multicolumn{2}{|l|}{ Total number } & \multirow{2}{*}{$\frac{\mathrm{V}-\mathrm{n}=16}{13(81 \%)}$} & \multirow{2}{*}{$\begin{array}{l}\mathrm{V}+\mathrm{n}=09 \\
7(78 \%)\end{array}$} & \multirow{2}{*}{$\frac{p \text { value }}{1.00^{\#}}$} & \multirow{2}{*}{$\begin{array}{l}\mathrm{V} 1 \mathrm{n}=04 \\
3(75 \%)\end{array}$} & \multirow{2}{*}{$\frac{V 2 n=05}{4(80 \%)}$} \\
\hline Olfactory dysfunction & Overall incidence & & & & & \\
\hline & Hyposmia & $6(46 \%)$ & 0 & $0.05^{\#}$ & 0 & 0 \\
\hline & Anosmia & $7(54 \%)$ & $7(100 \%)$ & & $3(100 \%)$ & $4(100 \%)$ \\
\hline & Median total duration, in days (range) & $32.8(5-88)$ & $28(12-52)$ & $0.78 *$ & $29(17-48)$ & $19.5(12-52)$ \\
\hline \multirow[t]{4}{*}{ Gustatory dysfunction } & Overall incidence & $13(81 \%)$ & $8(89 \%)$ & $1.00^{\#}$ & $4(100 \%)$ & $4(80 \%)$ \\
\hline & Hypogeusia & $11(84.6 \%)$ & $6(75 \%)$ & $0.61^{\#}$ & $3(75 \%)$ & $3(75 \%)$ \\
\hline & Ageusia & $2(15.4 \%)$ & $2(25 \%)$ & & $1(20 \%)$ & $1(25 \%)$ \\
\hline & Median total duration in days (range) & $16(1-35)$ & $15(2-29)$ & $0.74 *$ & $16(2-29)$ & $12.5(2-23)$ \\
\hline
\end{tabular}

V1- participants who had received single dose of vaccine

V2- participants who had received two doses of vaccine

\#Fisher's exact test, *Wilcoxon ranksum test

\section{Results}

This cohort of 25 otolaryngologists, included 17 males and 8 females in their $3^{\text {rd }}-4^{\text {th }}$ decade of life. Considering two weeks as the minimum duration to develop antibodies following vaccination, doctors who reported symptoms within 2 weeks of the first dose of vaccination were classified as unvaccinated $(\mathrm{V}+)$. Accordingly, 16 physicians were unvaccinated $(\mathrm{V}-), 04$ vaccinated with single dose (V1) and 05 with complete course of two doses of vaccine (V2). The median duration between vaccination and symptom onset was 32 days (range 5-73 days). 24 of 25 physicians had mild disease and one unvaccinated physician developed moderate illness. The incidence of loss of smell was $80 \%$ and loss of taste was $84 \%$. Onset of chemosensory loss was in the first week of illness in all of them. Complete recovery of olfactory and gustatory disturbances was observed by 4 weeks in $70 \%(\mathrm{n}=14)$ and 95\% ( $\mathrm{n}=20)$ of them respectively. Except one physician whose anosmia improved to subjective mild hyposmia over a span of 85 weeks, rest of them had complete recovery. The details of the chemosensory disturbances noted in the $\mathrm{V}-$ and $\mathrm{V}+$ group are presented in Table 1 .

\section{Discussion}

This observation based on a small cohort of otolaryngologists, who provided highly reliable and precise features of their symptomatology, showed similar incidence of chemosensory disturbances in the unvaccinated and vaccinated group. However, higher rate anosmia and ageusia was reported in breakthrough COVID-19 illness. Systemic symptoms in breakthrough COVID-19 illness have been reported as of lesser severity as compared to primary COVID-19 infection due to the protection provided by vaccine induced antibodies. However, circulating antibodies might not provide protection to the virus targeted chemosensory receptors and nerves located in the olfactory cleft as well as oral cavity. This may explain the lack of less severe chemosensory loss in breakthrough COVID-19. Recovery from chemosensory loss is fastened by the circulating antibodies as indicated by lesser total duration of dysfunction in the V2 group as compared to V- group. Any definite observational remark on the pattern of chemosensory loss seemed unwarranted between the partially (V1, $\mathrm{n}=4$ ) and completely (V2, $\mathrm{n}=5$ ) vaccinated groups due to small sample size. To find out the significance of the results noted in this small series, larger population-based studies and use of objective smell and taste tests should be considered.

Author contribution PS: Data collection, Analysis, Writing the paper. RK: Analysis, Writing the paper, AT: Analysis, Writing the paper.

\section{Declarations}

Conflict of interest None.

Ethics Approval Study approved by Institute ethics committee, AIIMS, New Delhi.

Human or Animal participants Yes.

Informed consent Yes.

\section{References}

1. Teran RA, Walblay KA, Shane EL, et al. Postvaccination SARSCoV-2 Infections Among Skilled Nursing Facility Residents and Staff Members - Chicago, Illinois, December 2020-March 2021. MMWR Morb Mortal Wkly Rep. 2021;70(17):632-638. 
Published 2021 Apr 30. doi:https://doi.org/10.15585/mm wr.mm7017e1.

2. Tyagi K, Ghosh A, Nair D et al (2021) Breakthrough COVID19 infections after vaccinations in healthcare and other workers in a chronic care medical facility in New Delhi India. Diabetes Metab Syndr 15(3):1007-1008. https://doi.org/10.1016/j.dsx.2021.05.001

3. Sagar P, Kumar R, Fatima R, et al. Prospective Analysis of the Chronology of Smell and Taste Dysfunction in COVID-19
Positive ENT Doctors [published online ahead of print, 2021 Apr 23]. Indian J Otolaryngol Head Neck Surg. 2021;1-6.

Publisher's Note Springer Nature remains neutral with regard to jurisdictional claims in published maps and institutional affiliations. 